\title{
Plasmodium malariae in Chittagong, Bangladesh
}

\author{
Muhammad Ehsanul Haque1, Muhammad Anwar Husain1, Nasima A kter'1, Muhammad A bdul Mazed1, K han Mashrequl Alam1, \\ Muhammad Tipu Sultan1, A rup Kanti Dewanjee1, Muhammad Shaqil Ahmed1, Muhammad A rifur Rahman1, Muhammad Zakir Hossain1 \\ 1 Departmetn of Microbiology, Chittagong Medical College, Chittagong
}

\section{Summary:}

We detected a case of Plasmodium malariae in Chittagong, Bangladesh. The person was an adult male, presented clinically as severe malaria. Blood slide examination by microscopy and pan malarial immunochromatographic test (ICT) revealed the case as Plasmodium malariae infection. The patient presented as severe malaria case for which he was hospitalized and recovered after seven days of quinine treatment.

\section{Background:}

Malaria remains a global concern. An estimated 300-500 million clinical cases occur each year throughout the world. A mong them deaths occur in between 1.1-2.7 millions, most of them are children of below 5 years of age. $V$ ast majority of cases are found in 107 countries in the tropical regions of A frica, A sia, Central and South A mericas where the disease is endemic ${ }^{1,2}$.

Malaria is also a major public health concern in B angladesh. A pproximately $33.6 \%$ of total populations ( 150 million) of the country are at risk of malaria. So far two species of malarial parasites i.e., $P$ vivax and $P$ falciparum are prevalent in the country but there is evidence of increasing proportion of malaria cases by $\mathrm{P}$ falciparum which become severe and often complicated ${ }^{2}$. A part from those two species recently $\mathrm{P}$ malariae is being detected which presents with severe disease.

\section{Case report:}

Md A bu Bakar, a 45 yr old male from A nwara in Chittagong got himself admitted in Chittagong Medical College Hospital $(\mathrm{CMCH})$ in January, 2011. On admission clinical presentations as stated by the patient were high fever with rigor for one week associated with severe headache, bodyache, anorexia and prostration. At first there was evening rise of temperature with rigor which became

\footnotetext{
Correspondence:

Dr. Muhammad Ehsanul Haque

Lecturer,

Departmetn of Microbiology,

Chittagong Medical College, Chittagong

Mob: 01819375563

E-mail: ehkajol@yahoo.com
}

continuous in nature after three days. Having no definite diagnosis and physical improvement within short period of time patient himself left the hospital and visited a local physician who sent him to a local clinical laboratory asking for a blood examination for detection of malaria parasite with other relevant investigations. Blood smear microscopy of thick film revealed the presence of malaria parasite and the thin film showed $P$ malariae of trophozoite form. Subsequent ICT Pf/Pv showed negative result. On the next day clinical investigations were again done in the dept of Microbiology of Chittagong Medical College for further confirmation. The thin blood film showed malaria parasite in trophozoite form with typical band form and merozoites. Only mature RBC was infected with no change in RBC size. Malaria parasite count found to be $550 / \mathrm{mm}^{3}$ of blood. ICT Pf/Pv (SD BIOLINE) again showed negative result whereas ICT Pan/Pf/Pv (Zephyr Biomedicals, Goa, India) was found positive which indicated the species were either $P$ malariae or $\mathrm{P}$ ovale. Other laboratory parameters such as Haemoglobin, total and differential count of WBC, platelet count, serum creatinine and serum bilirubin were normal. Blood smear microscopy of malaria parasite with definite morphological resemblance with $\mathrm{P}$ malariae and a positive ICT Pan/Pf/Pv assay of same blood sample established the diagnosis as $P$ malariae infection.

The patient was treated with oral quinine therapy and recovered rapidly.

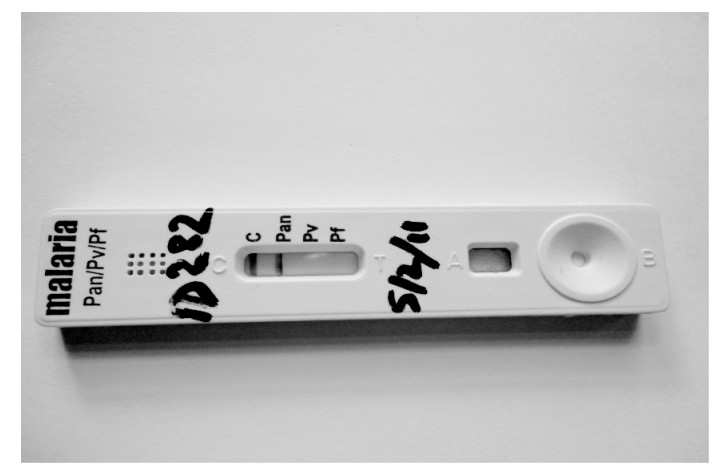

Fig I: I mmunochromatographic test -positive for $P$. malaria/ovale 


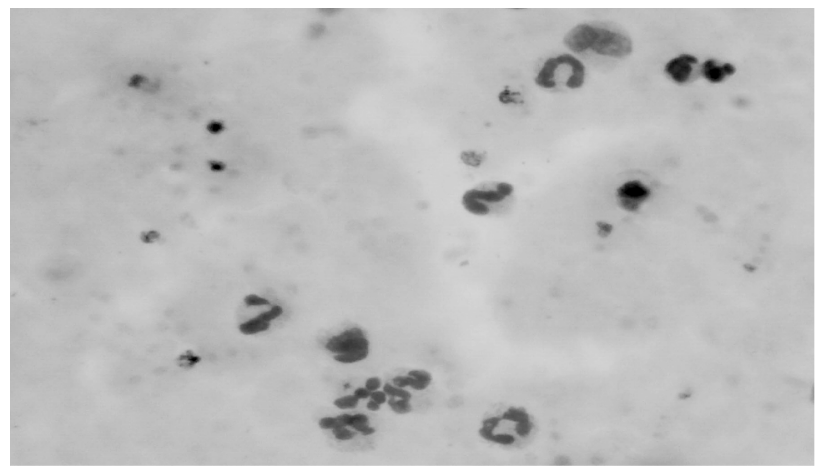

Fig II: Showing P. malariae in thick film

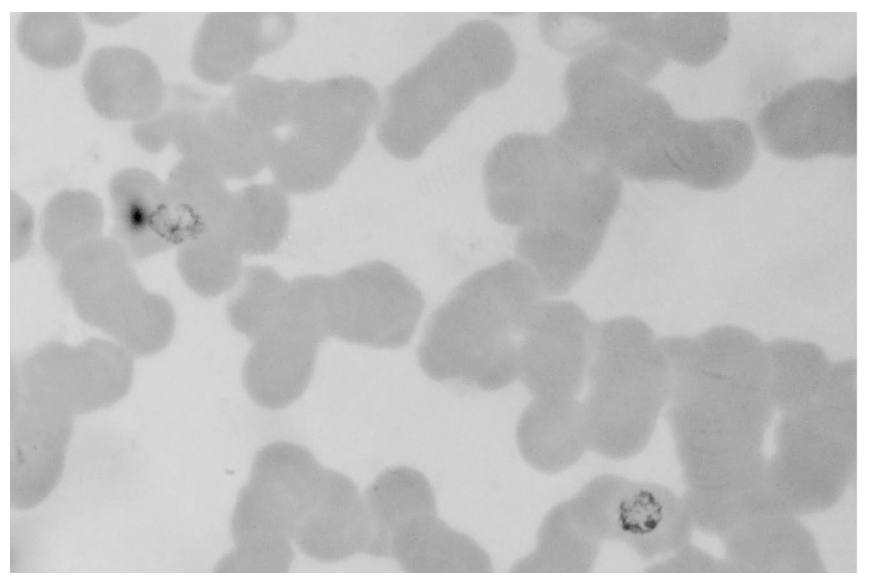

Fig III: Showing P. malariae in thin film

\section{Discussion:}

P. falciparum (malignant tertian malaria) and P. malariae (quartan malaria) are the most common species found in A sia and $A$ frica $^{3}$, but P. malariae infection has been reported rarely from Bangladesh ${ }^{4}$. So far only one case of $P C R$ confirmed $P$. malariae infection has been reported from Bangladesh ${ }^{4}$. The present case has been diagnosed with the help of microscopy and ICT Pan/Pf/Pv assay. There was a delay in parasitological diagnosis due to the fact that the clinical presentations were more severe and atypical unlike $P$. malariae infection and much used ICT Pf/Pv assay was found negative. The classic quarternary pattern of fever for $P$. malariae infection was absent.

We performed these laboratory examinations in a tertiary care centre where laboratory set up and experienced microbiologists are available otherwise there is every chance of missing those cases if proper procedure has not been followed as early stages of $P$. malariae could be difficult to distinguish from other species by microscopy alone. In such kind of situation ICT Pan/Pf/Pv assay could be a good alternative in addition to microscopic examination.

In summary, we report a rare case of $P$. malariae infection with severe illness. The patient made a rapid recovery with 7 days of oral quinine therapy.

\section{Conclusion:}

Though P. malariae is uncommon in this area but not absent. Proper laboratory diagnosis by trained personnel and newer immunochromatography kit may reveal the actual scenario. Moreover proper guideline for management of those rare malaria species at national level should be taken to avoid fatal complications.

\section{R efer ences:}

1. Mangold,K.A, Manson R.U, Koay S.C, Stephens L, Regner M.A, Thomson B.R., Peterson R.L, Kaul K.L. Real -Time PCR for detection and identification of plasmodium species. J. Clin. Micro. 2005; 43:2435-2440.

2. World Health Organization, Global burden of malaria WHO, 2005 [online]. [viewed31/10/2007]. [Webhttp://www.unmillenniumproject. org/documents/ Global B urden of Malaria.pdf]

3. Ghaffer A,[online] Medical Microbiology and Immunology, University of South Carolina , [viewed1/03/2011].web http://pathmicro.med.sc.edu/ parasitology/blood-proto.htm].

4. W. Rahman, K. Chotivanich, K. Silamut, N. Tanomsing, A. Hossain, M.A. Faiz, A.M. Dondorp, and R.J. Maude, 2010 Plasmodium malariae in B angladesh, Trans $\mathrm{R}$ Soc Trop Med Hyg; 104(1): 78-80. 